\title{
Strong Agreement Between Magnetic Resonance Imaging and Radiographs for Caton-Deschamps Index in Patients With Patellofemoral Instability
}

\author{
Ryan W. Paul, B.S., Joseph M. Brutico, B.S., Margaret L. Wright, M.D., \\ Brandon J. Erickson, M.D., Fotios P. Tjoumakaris, M.D., Kevin B. Freedman, M.D., and \\ Meghan E. Bishop, M.D.
}

\begin{abstract}
Purpose: To compare the measurements of the Caton-Deschamps index on preoperative magnetic resonance imaging and radiographs of patients undergoing operative management of patellar instability. Methods: Patients who underwent primary medial patellofemoral ligament reconstruction and/or tibial tubercle osteotomy between January 2015 and November 2019 were assessed. Caton-Deschamps indices were measured by 3 independent reviewers on both radiographs and magnetic resonance imaging. Intra- and interclass correlation coefficients and a Bland-Altman analysis were calculated to assess inter-rater reliability and measurement agreement between radiographic and magnetic resonance imaging. Results: Seventy-two patients (73 knees) were identified. The average Caton-Deschamps index was $1.23 \pm 0.18$ on radiograph and $1.26 \pm 0.18$ on magnetic resonance imaging. Strong inter-rater reliability was observed between reviewers for both radiographic and magnetic resonance imaging Caton-Deschamps indices (intraclass correlation coefficients 0.700 and 0.715 , respectively). Pooled observer measurements revealed a moderate agreement between radiographic and magnetic resonance imaging for patella to tibia distance, weak agreement for patellar articular cartilage distance, and strong agreement for the Caton-Deschamps index (intraclass correlation coefficients $0.687,0.485$, and 0.749, respectively). Bland-Altman analysis demonstrated a mean difference in Caton-Deschamps index of $-0.03 \pm 0.15(95 \%$ limits of agreement: -0.29 to 0.23 ) between radiographic and magnetic resonance imaging, meaning that Caton-Deschamps indices were on average 0.03 lower on radiographic than on magnetic resonance imaging. Conclusions: The Caton-Deschamps index has strong agreement between radiographic and magnetic resonance imaging in patients undergoing patellar stabilization surgery. Either modality can be reliably used to preoperatively assess patellar height. Level of Evidence: Level IV, diagnostic case series.
\end{abstract}

$\mathbf{P}$ atellar instability is a common condition found in sports medicine offices, with an incidence of 23 per 100,000 persons in the general population and 148 per 100,000 persons in adolescents aged 14 to 18 years. ${ }^{1}$ Recent years have seen an increase in patellar

From the Rothman Orthopaedic Institute, Philadelphia, Pennsylvania (R.W.P., J.M.B., M.L.W. F.P.T., K.B.F.); and Rothman Orthopaedic Institute, New York, New York (B.J.E., M.E.B.), U.S.A.

The authors report the following potential conflicts of interest or sources of funding: B.J.E. reports board or committee member for American Orthopaedic Society for Sports Medicine (AOSSM), American Academy of Orthopaedic Surgeons (AAOS), and American Shoulder and Elbow Surgeons (ASES), consultant for Arthrex, DePuy, Linvatec, Smith $\theta$ Nephew, and Stryker; and editorial or governing board for PLOS One, outside the submitted work. F.P.T. reports board or committee member for AOSSM, AAOS, and ASES; and stock options from Franklin/Keystone Biosciences and Trice Medical, outside the submitted work. K.B.F. reports board or committee member for AOSSM; consultant for DePuy and Vericel; and educational presentations for Liberty Surgical, outside the submitted work. M.E.B. reports board or committee member for AOSSM and AAOS, outside the submitted work. Full ICMJE instability, as 86.0 children per hospital were reported with patellar dislocations in 2016 compared with only 14.5 children per hospital in $2004 .^{2}$ The etiology of patellar instability is multifactorial, with many patients having predisposing anatomic factors that alter the

author disclosure forms are available for this article online, as supplementary material.

Primary institution where this investigation was performed: Rothman Orthopaedic Institute, Philadelphia, Pennsylvania, U.S.A.

Received March 24, 2021; accepted July 24, 2021.

Address correspondence to Meghan E. Bishop, M.D., Assistant Professor of Orthopaedic Surgery, Rothman Orthopaedic Institute, 645 Madison Ave., New York, NY 10022.E-mail: meghan.bishop@rothmanortho.com

(C) 2021 THE AUTHORS. Published by Elsevier Inc. on behalf of the Arthroscopy Association of North America. This is an open access article under the CC BY-NC-ND license (http://creativecommons.org/licenses/by-nc-nd/4.0/). 2666-061X/21456

https://doi.org/10.1016/j.asmr.2021.07.017 


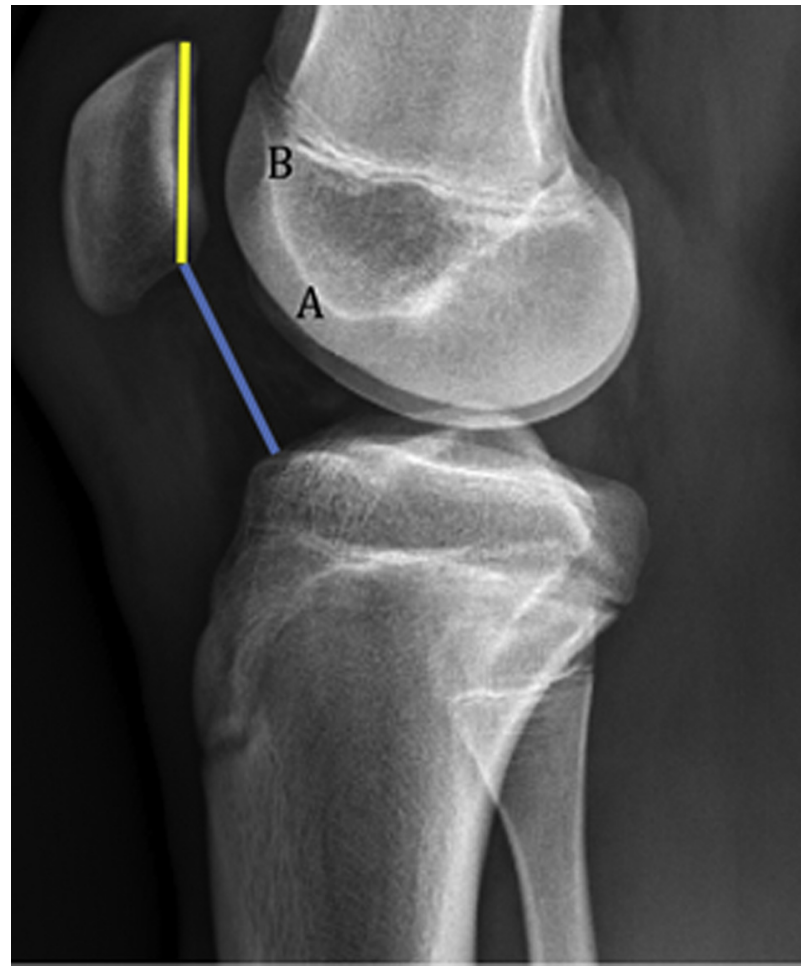

Fig 1. CDI measurement from lateral radiograph with knee at $30^{\circ}$ of flexion. $\mathrm{CDI}=\mathrm{A} / \mathrm{B}$, the distance between the anterosuperior point of the tibial plateau and the distal pole of the patellar articular surface (A: blue line), divided by the articular cartilage length of the patella (B: yellow line). (CDI, Caton-Deschamps index.)

biomechanics of the patellofemoral joint, including trochlear dysplasia, limb malalignment, and patella alta. $^{3-10}$

Patients with patella alta have a 4 -fold increase in recurrent patellar dislocations compared with control patients. ${ }^{11}$ This is due to the fact that the patella does not engage in the trochlear groove until a high knee flexion angle in patients with patella alta, thereby increasing the risk of instability. ${ }^{10,12,13}$ Hence, measurement of patellar height is an important technique for evaluating the risk of patellar instability and determining subsequent treatment options. ${ }^{11,14-16}$ There are a number of methods to radiographically measure patellar height, including the Insall-Salvati (IS), modified Insall-Salvati (MIS), Caton-Deschamps Index (CDI), and Blackburne-Peel (BP). ${ }^{17-20} \mathrm{~A}$ metaanalysis performed by Smith et al. $^{20}$ evaluated the reliability and validity of various patellar height measurements, finding good reliability and validity for 7 different methods with no method standing out as clinically superior.

Yue et al. ${ }^{21}$ investigated IS, MIS, CDI, and BP measurements on both lateral radiographic and magnetic resonance imaging (MRI) in patients with lateral patellar dislocation and compared these measurements with a control group. The authors found that the patellar height of the lateral patellar dislocation group was measured greater on MRI than radiograph for all 4 measurements, and patellar height was also greater on MRI in the control group for CDI and BP ratios. ${ }^{21}$ Furthermore, Verhulst et al. ${ }^{22}$ investigated intra- and inter-rater reliability on radiographs, computed tomography, and MRI in 48 patients presenting with patellar instability. Interestingly, the CDI showed good reliability on radiographs, moderate reliability on computed tomography, and poor reliability on MRI. ${ }^{22}$ However, in a 2017 study that included a survey of the members of the International Patellofemoral Study Group, consensus established the CDI as the preferred method for measuring patellar height. ${ }^{23}$ The CDI has been considered the most functional assessment of patellar height and provides great clinical advantage due to the prevalence of lateral radiographs and the simplicity of CDI measurement. ${ }^{24}$ Also, unlike the IS ratio, CDI is not affected by concomitant tibial tuberosity abnormalities, allowing for a more universal clinical application.

Measurement of the CDI can be determined on both radiographs and MRI. ${ }^{25}$ Lateral knee radiographs are often taken in a weight-bearing position with the knee at $30^{\circ}$ of flexion, whereas MRI is taken with the patient in the supine position with the knee in extension. Variability in knee positioning and imaging modality

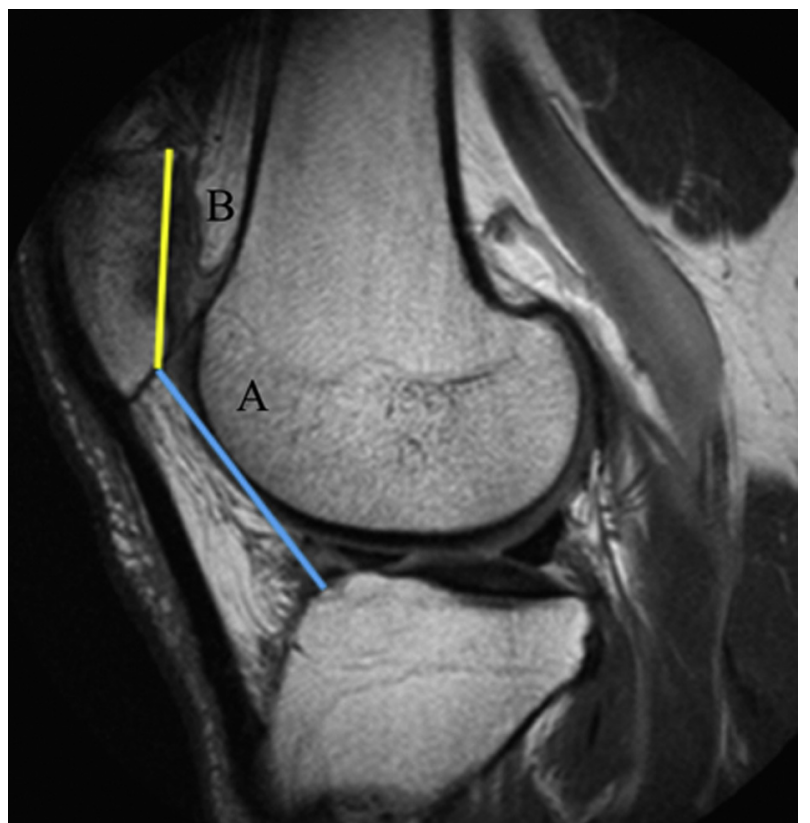

Fig 2. CDI measurement from sagittal proton density-weighted magnetic resonance imaging slice with the greatest length of the patella. $\mathrm{CDI}=\mathrm{A} / \mathrm{B}$, the distance from the distal aspect of the patellar articular cartilage to the anterosuperior corner of the tibial joint surface (A: blue line), divided by the length of the cartilaginous articular surface (B: yellow line). (CDI, Caton-Deschamps index.) 
Total patients screened for inclusion

$$
(\mathrm{n}=881)
$$

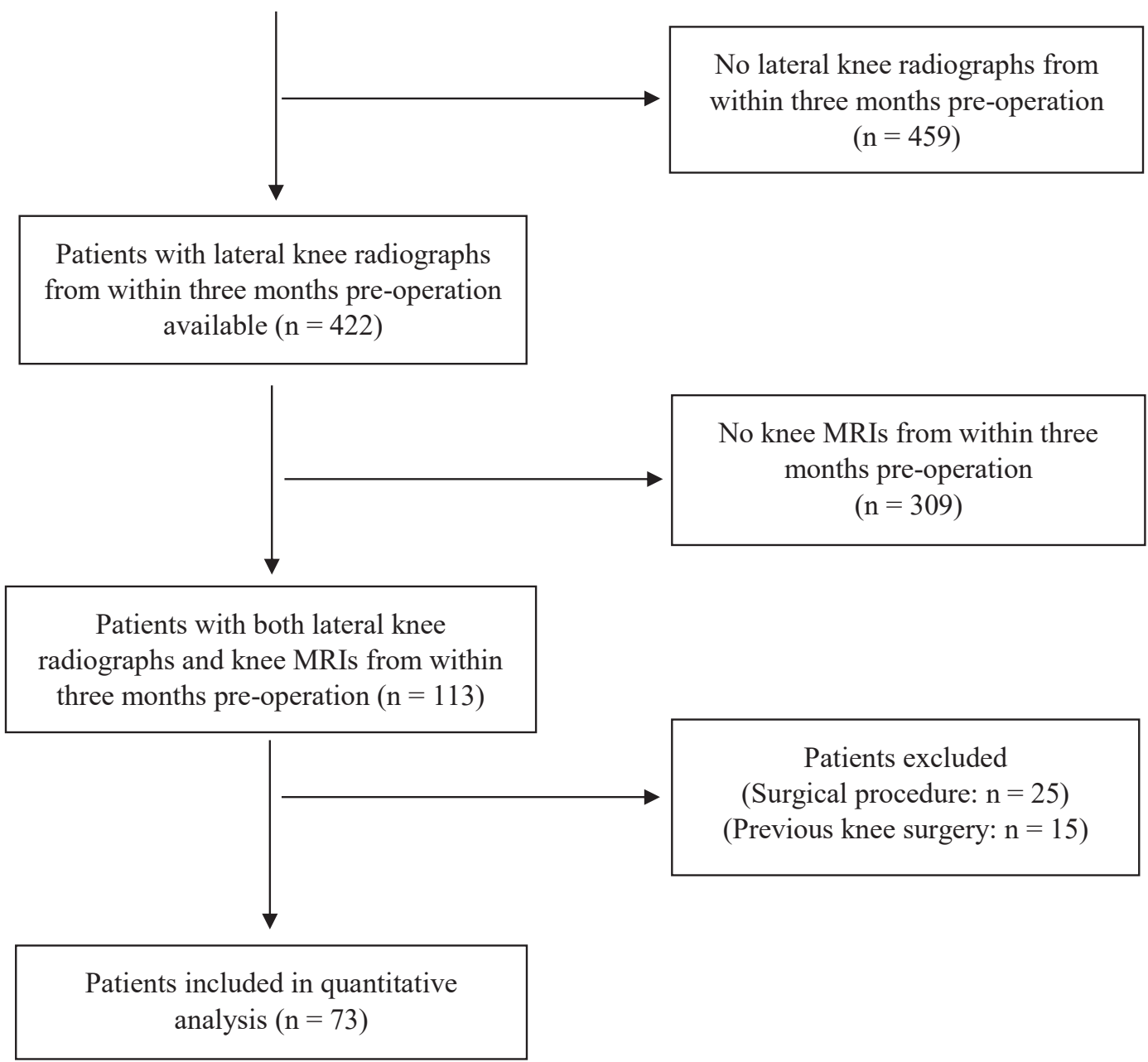

Fig 3. Flow chart of patient screening and final quantitative analysis. (MRI, magnetic resonance imaging.)

may affect patellar height and CDI measurement. ${ }^{26}$ While previous studies have examined the inter-rater reliability of the CDI for measurement of patellar height, the agreement between measurements from radiographs and MRI remains unclear. Evaluating the reliability and agreement between imaging modalities can help determine the clinical applicability of each modality, potentially allowing clinicians to simply use standard-of-care imaging instead of requiring further imaging. The purpose of this study was to compare the measurements of the CDI on preoperative MRI and radiographs of patients undergoing operative management of patellar instability. The null hypothesis was that there would be no difference in CDI between imaging modalities.

\section{Methods}

Study approval was obtained from the institutional review board (study \#19E.938 was exempt from
Jefferson University institutional review board review on December 19, 2019). A list of all patients who underwent medial patellofemoral ligament reconstruction (MPFLR) and/or tibial tubercle osteotomy (TTO) from

Table 1. Baseline Characteristics of Included Patients

\begin{tabular}{lc}
\hline \multicolumn{1}{c}{ Characteristics } & Patients \\
\hline Female sex (\% female) & $41(56.9 \%)$ \\
Age at surgery, y & $25.4 \pm 11.1(11.9-56.3)$ \\
Surgical procedure & $49(67.1 \%)$ \\
MPFLR only & $5(6.8 \%)$ \\
TTO only & $19(26.0 \%)$ \\
Both MPFLR and TTO & $29(39.7 \%)$ \\
Laterality & $44(60.3)$ \\
Right & \\
Left & \\
NOTE. Sex, surgical procedure, and laterality presented as $\mathrm{n}(\%)$, \\
and age at surgery presented as mean \pm standard deviation (range). \\
MPFLR, medial patellofemoral ligament reconstruction; TTO, tibial \\
tubercle osteotomy.
\end{tabular}


Table 2. Average Caton-Deschamps Index (CDI), Patella to Tibia Distance, and Patellar Articular Cartilage Distance, Among Each Investigator for Radiographs and MRI

\begin{tabular}{|c|c|c|c|c|c|c|c|c|}
\hline \multirow[b]{2}{*}{ Variable } & \multicolumn{2}{|c|}{ Investigator 1} & \multicolumn{2}{|c|}{ Investigator 2} & \multicolumn{2}{|c|}{ Investigator 3} & \multicolumn{2}{|c|}{ Total $(\mathrm{n}=219)$} \\
\hline & Radiograph & MRI & Radiograph & MRI & Radiograph & MRI & Radiograph & MRI \\
\hline$\overline{\mathrm{CDI}}$ & $\begin{array}{l}1.23 \pm 0.16 \\
(1.19-1.26)\end{array}$ & $\begin{array}{l}1.23 \pm 0.16 \\
(1.19-1.27)\end{array}$ & $\begin{array}{l}1.20 \pm 0.19 \\
(1.16-1.24)\end{array}$ & $\begin{array}{l}1.28 \pm 0.20 \\
(1.23-1.33)\end{array}$ & $\begin{array}{l}1.25 \pm 0.18 \\
(1.21-1.29)\end{array}$ & $\begin{array}{l}1.26 \pm 0.17 \\
(1.23-1.30)\end{array}$ & $\begin{array}{l}1.23 \pm 0.18 \\
(1.20-1.25)\end{array}$ & $\begin{array}{l}1.26 \pm 0.18 \\
(1.24-1.28)\end{array}$ \\
\hline $\begin{array}{l}\text { Patella to tibia } \\
\text { distance, mm }\end{array}$ & $\begin{array}{l}43.33 \pm 6.05 \\
(41.95-44.72)\end{array}$ & $\begin{array}{l}39.50 \pm 5.17 \\
(38.32-40.69)\end{array}$ & $\begin{array}{l}43.36 \pm 6.46 \\
(41.87-44.84)\end{array}$ & $\begin{array}{l}40.69 \pm 5.43 \\
(39.45-41.94)\end{array}$ & $\begin{array}{l}43.64 \pm 6.68 \\
(35.76-42.11)\end{array}$ & $\begin{array}{l}39.35 \pm 5.03 \\
(38.20-40.51)\end{array}$ & $\begin{array}{l}43.44 \pm 6.37 \\
(42.60-44.29)\end{array}$ & $\begin{array}{l}39.85 \pm 5.22 \\
(39.16-40.54)\end{array}$ \\
\hline $\begin{array}{l}\text { Patellar articular } \\
\text { cartilage } \\
\text { distance, mm }\end{array}$ & $\begin{array}{l}35.44 \pm 3.72 \\
(34.59-36.29)\end{array}$ & $\begin{array}{l}32.29 \pm 3.75 \\
(31.43-33.15)\end{array}$ & $\begin{array}{l}36.35 \pm 3.59 \\
(35.52-37.17)\end{array}$ & $\begin{array}{l}32.05 \pm 3.31 \\
(31.29-32.81)\end{array}$ & $\begin{array}{l}35.05 \pm 3.08 \\
(34.35-35.76)\end{array}$ & $\begin{array}{l}31.28 \pm 3.24 \\
(30.54-32.03)\end{array}$ & $\begin{array}{l}35.61 \pm 3.50 \\
(35.15-36.08)\end{array}$ & $\begin{array}{l}31.88 \pm 3.45 \\
(31.42-32.33)\end{array}$ \\
\hline
\end{tabular}

NOTE. Data presented as mean \pm standard deviation (95\% confidence interval).

MRI, magnetic resonance imaging.

January 1, 2015, to November 30, 2019, were obtained from the medical records of a single institution. Patients with both a preoperative $30^{\circ}$ standing lateral radiograph and a sagittal proton density-weighted MRI from within 3 months of surgery were included in the study. Patients were excluded from the study if they did not undergo MPFLR and/or TTO, or if they underwent a previous ipsilateral knee surgery. In addition, patients were excluded if they were without both a preoperative radiograph and MRI, or if imaging had occurred greater than 3 months before surgery.

Patient sex, age, laterality, and procedure date were collected from clinical records. Three independent reviewers, including an attending sports medicine surgeon (M.E.B.), a sports medicine clinical fellow (M.L.W.), and a trained research assistant (R.W.P.), measured the CDI on radiographic and MRI images for all included patients. To measure the CDI on radiograph, the technique previously described by Caton et al. ${ }^{22,27}$ was used, as the ratio of the distance between the anterosuperior point of the tibial plateau and the distal pole of the patellar articular surface to the articular surface length of the patella (Fig 1). The CDI on MRI was determined via the technique described by Askenberger et al., ${ }^{28}$ with the sagittal slice showing the greatest length of the patella (through the central part of the patellar facet) used for sagittal measurements of patellar height (Fig 2).

Inter-rater reliability and agreement between measurement modalities (radiographs and MRI) was determined by calculating the intraclass correlation coefficient (ICC). ${ }^{22}$ The ICC scores were interpreted as follows: a score $<0.50$ indicating weak reliability, 0.50 to 0.69 indicating moderate reliability, and a score of 0.70 to 1.00 indicating strong reliability. Inter-rater reliability was evaluated for both distance (patella to tibia distance and patellar articular cartilage distance) measures of the CDI, as well as the CDI itself, on both MRI and radiographs. Since strong inter-rater reliability was observed, data from the 3 investigators were pooled for an overall assessment of MRI versus radiograph agreement. This pooled assessment of MRI versus radiograph was performed with ICCs and Bland-Altman 95\% limits of agreement. The Student $t$ test was used to examine the difference in mean CDI on radiographs and MRI, with statistical significance set at .05.

\section{Results}

After we screened inclusion and exclusion criteria, a final cohort of 72 patients (73 knees) was available for quantitative analysis (Fig 3). There were 41 female $(56.9 \%)$ and 31 male $(43.1 \%)$ patients evaluated, with a mean age of $25.4 \pm 11.1$ years (range, 11.9-56.3 years) (Table 1 ).

Patella to tibia distance and patellar articular cartilage distance were longer on radiograph than on MRI for each observer, although the difference was not statistically significant $(P=.200)$ (Table 2$)$. The average CDI was $1.23 \pm 0.18$ (95\% confidence interval 1.20-1.25) on radiograph and $1.26 \pm 0.18(95 \%$ confidence interval 1.24-1.28) on MRI. There was moderate interrater reliability for the measurement of patellar

Table 3. Inter-rater Reliability of Each Caton-Deschamps Index (CDI) Measurement Between Three Investigators

\begin{tabular}{lccc}
\hline \multicolumn{1}{c}{ Variable } & ICC Value & 95\% Confidence Interval & Reliability \\
\hline Patella to tibia radiograph & 0.923 & $0.890-0.949$ & Strong \\
Patella to tibia MRI & 0.906 & $0.842-0.943$ & Strong \\
Articular cartilage radiograph & 0.679 & $0.561-0.776$ & Moderate \\
Articular cartilage MRI & 0.752 & $0.655-0.829$ & Strong \\
CDI radiograph & 0.700 & $0.594-0.788$ & Strong \\
CDI MRI & 0.715 & $0.612-0.800$ & Strong \\
\hline
\end{tabular}

ICC, intra-class correlation coefficient; MRI, magnetic resonance imaging. 
Table 4. Intra-rater Agreement Between Radiographs and MRI

\begin{tabular}{lccc}
\hline \multicolumn{1}{c}{ Variable } & ICC Value & 95\% Confidence Interval & Agreement \\
\hline Investigator l & & & \\
$\quad$ Patella to tibia & 0.659 & $0.033-0.861$ & Moderate \\
Articular cartilage distance & 0.575 & $0.000-0.824$ & Moderate \\
CDI & 0.797 & $0.694-0.867$ & Strong \\
Investigator 2 & & & Strong \\
$\quad$ Patella to tibia & 0.700 & $0.391-0.840$ & No true agreement \\
Articular cartilage distance & 0.015 & $0.001-0.032$ & Moderate \\
CDI & 0.543 & $0.321-0.700$ & Moderate \\
Investigator 3 & 0.615 & $0.020-0.834$ & Weak \\
$\quad$ Patella to tibia & 0.383 & $0.000-0.696$ & Moderate \\
$\quad$ Articular cartilage distance & 0.593 & $0.422-0.723$ & \\
CDI & & &
\end{tabular}

CDI, Caton-Deschamps index; ICC, intraclass correlation coefficient.

articular cartilage on radiograph (Table 3). However, patellar articular cartilage measurements on MRI demonstrated strong inter-rater reliability. CDI measurement on both radiograph and MRI also showed strong inter-rater reliability. Specifically, patella to tibia measurements on both radiograph and MRI showed very strong reliability with ICC values greater than 0.900 .

Moderate agreement between radiograph and MRI CDIs was observed for 2 investigators, and strong agreement between radiograph and MRI CDIs was observed for 1 investigator (Table 4). Because there was strong inter-rater reliability, data from the various investigators were able to be pooled into one combined assessment of MRI versus radiograph. Overall, there was moderate agreement between radiograph and MRI for patella to tibia distance, weak agreement for patellar articular cartilage distance, and strong agreement for the CDI (Table 5). Bland-Altman analysis demonstrated a mean difference in CDI of $-0.03 \pm 0.15(95 \%$ limits of agreement: -0.29 to 0.23 ) between radiographs and MRI, meaning that CDIs were on average 0.03 lower on radiographs than on MRI (Fig 4).

\section{Discussion}

This study found strong agreement between CDI measurements from radiographs and MRI. Both imaging modalities also showed strong inter-rater reliability between 3 independent investigators. Overall, this study suggests that both imaging modalities can be reliably used for measuring patella alta with the CDI.

Current literature has shown inconsistent results regarding the reliability of measuring CDI, as CDI measurements have ranged from poor to excellent on radiograph and MRI. ${ }^{21,22,29-35}$ The discrepancy of reported inter-rater reliability may be attributed to several factors, such as differences in identification of bony landmarks, radiographic measurements amongst observers, criteria used for categorizing patellar height, and the statistical methods implemented for classifying reliability in prior studies. While other measurements of patellar height offer alternatives to the CDI (IS, MIS, BP, patellotrochlear index, sagittal patellofemoral engagement, and patellar articular overlap), some of these measurements do not have as well-established ranges of normal, may be affected by tibial tuberosity abnormalities, and are not as commonly used in a clinical setting. . $3,33,36-39$

Despite strong agreement for the overall CDI ratio between radiograph and MRI, weak agreement was found between MRI and radiographs for articular cartilage distance measurement. The inter-rater reliability presented in this current study suggests that clinicians can more accurately assess articular cartilage distance using MRI as opposed to radiograph, potentially due to the ability to visualize the articular cartilage on MRI. Due to poor visualization of the patellar articular cartilage on radiograph, clinicians may only estimate the true length of the cartilage resulting in imprecise and inconsistent measurements.

Decreased intra-rater agreement between MRI and radiograph may be partly attributed to the greater length of the patella and articular cartilage measured on radiograph compared with MRI. This finding is supported by similar reports in previous literature. ${ }^{21,40}$ In contrast, there are other sources of error from MRI that should not be ignored. MRI intra-rater reliability is highly dependent on not only the experience of the

Table 5. Pooled Agreement Between Radiographs and MRI Among Three Investigators

\begin{tabular}{lccc}
\hline \multicolumn{1}{c}{ Variable } & ICC Value & $\begin{array}{c}95 \% \text { Confidence } \\
\text { Interval }\end{array}$ & Agreement \\
\hline Patella to tibia & 0.687 & $0.076-0.872$ & Moderate \\
Articular cartilage & 0.485 & $0.000-0.803$ & Weak \\
CDI & 0.749 & $0.618-0.837$ & Strong \\
\hline
\end{tabular}

CDI, Caton-Deschamps index; ICC, intra-class correlation coefficient; MRI, magnetic resonance imaging. 


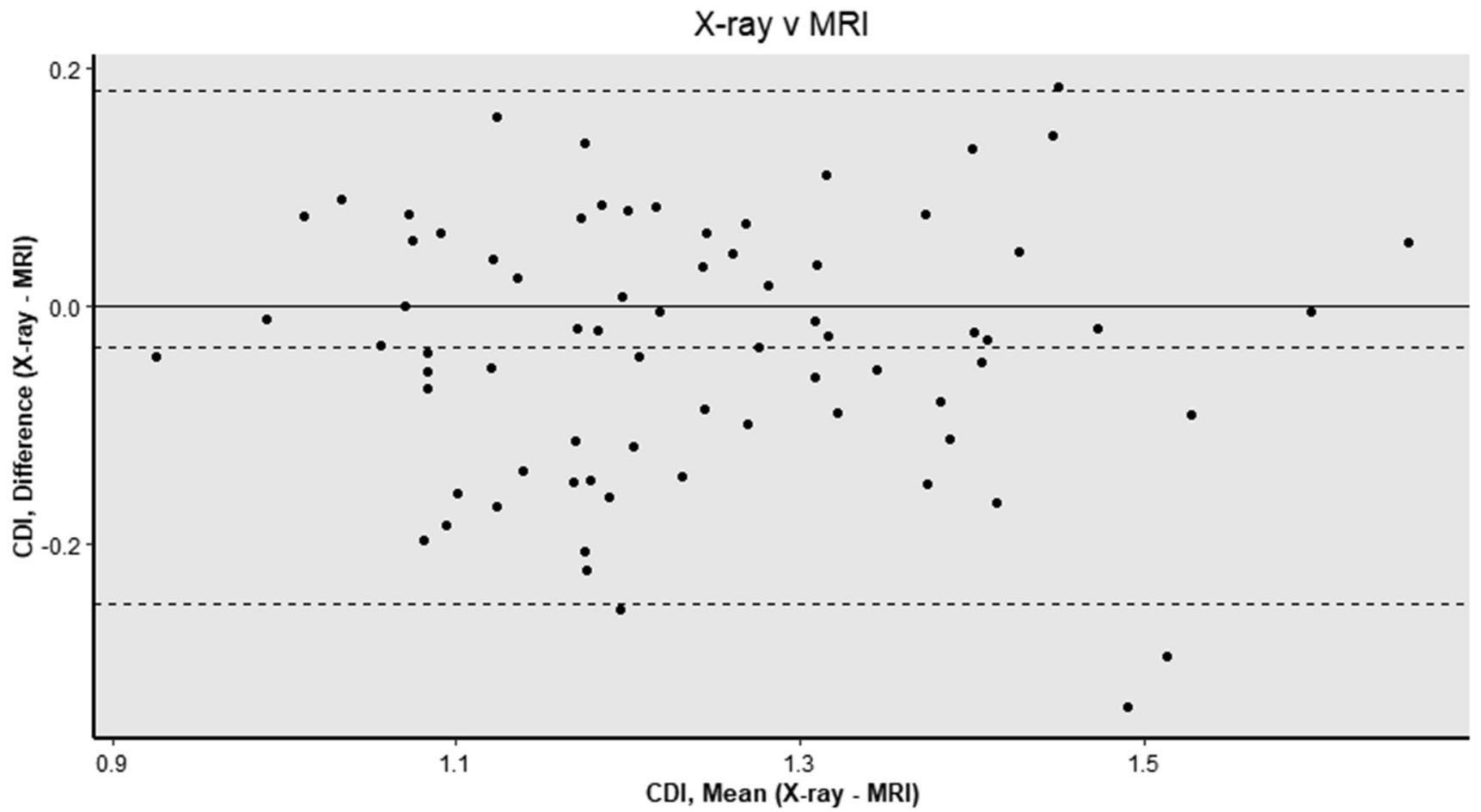

Fig 4. Bland-Altman analysis of agreement between radiographs and MRI. Top dashed line $=$ upper bound of the $95 \%$ limits of agreement, bottom dashed line = lower bound of the $95 \%$ limits of agreement, thin solid line $=$ CDI difference of zero, thick solid line $=$ the mean of -0.03 . (CDI, Caton-Deschamps index;(MRI, magnetic resonance imaging.)

observer but also on the sagittal MRI slice chosen during measurement. ${ }^{21,22,35}$ While the mid-sagittal slice is the most commonly used, ${ }^{29,41,42}$ Verhulst et al. ${ }^{22}$ argues that this slice infrequently provides the maximal measurement in regard to the length of the patella and articular cartilage in patients with patellar instability.

The findings of the present study are important due to its implications on surgical planning in patients diagnosed with patella alta. After failure of nonoperative management, patients undergoing MPFLR with patella alta are sometimes considered for a concomitant distalizing TTO to help with stability. While radiographs have traditionally provided visualization of the bony integrity and calculation of the CDI, MRI allows for visualization of not only bony, but also ligamentous and cartilaginous structures of the knee. The results of the current study suggest that MRI can provide a reliable CDI in addition to superior visualization of the structural integrity of the knee. While these results are encouraging, further examination of the reproducibility of the CDI on MRI is warranted due to the disagreements within existing literature.

\section{Limitations}

Radiographic imaging was of varying quality and therefore may have resulted in imperfect measurements. Also, lateral views of the knee are commonly taken with the knee in $30^{\circ}$ of flexion, but the amount of flexion could have varied among radiographs. To control for variability, 3 independent observers conducted measurements on all radiographic images. The differences in the experience of the observers may have led to measurement inconsistencies; however, all were trained in measuring CDI on both MRI and radiographs as described. Lastly, the CDI was calculated from radiographic images of patients who had undergone MPFLR and/or TTO, which likely skewed the data towards an average CDI larger than the general population. This may have led to increased reliability; however, measurement of CDI is most applicable to this patient population, so this effect is reflective of clinical applicability.

\section{Conclusions}

The CDI has strong agreement between radiograph and MRI in patients undergoing patellar stabilization surgery. Either modality can be reliably used to preoperatively access patellar height.

\section{References}

1. Sanders TL, Pareek A, Hewett TE, Stuart MJ, Dahm DL, Krych AJ. Incidence of first-time lateral patellar dislocation: A 21-year population-based study. Sports Health 2018;10:146-151.

2. Poorman MJ, Talwar D, Sanjuan J, Baldwin KD, Sutliff N, Franklin CC. Increasing hospital admissions for patellar instability: A national database study from 2004 to 2017. Phys Sportsmed 2020;48:215-221. 
3. Bartsch A, Lubberts B, Mumme M, Egloff C, Pagenstert G. Does patella alta lead to worse clinical outcome in patients who undergo isolated medial patellofemoral ligament reconstruction? A systematic review. Arch Orthop Trauma Surg 2018;138:1563-1573.

4. Dejour H, Walch G, Nove-Josserand L, Guier C. Factors of patellar instability: An anatomic radiographic study. Knee Surg Sports Traumatol Arthrosc 1994;2:19-26.

5. Ebinger TP, Boezaart A, Albright JP. Modifications of the Fulkerson osteotomy: A pilot study assessment of a novel technique of dynamic intraoperative determination of the adequacy of tubercle transfer. Iowa Orthop J 2007;27: 61-64.

6. Magnussen RA, De Simone V, Lustig S, Neyret P, Flanigan DC. Treatment of patella alta in patients with episodic patellar dislocation: A systematic review. Knee Surg Sports Traumatol Arthrosc 2014;22:2545-2550.

7. Sanchis-Alfonso V. Guidelines for medial patellofemoral ligament reconstruction in chronic lateral patellar instability. J Am Acad Orthop Surg 2014;22:175-182.

8. Wagner D, Pfalzer F, Hingelbaum S, Huth J, Mauch F, Bauer G. The influence of risk factors on clinical outcomes following anatomical medial patellofemoral ligament (MPFL) reconstruction using the gracilis tendon. Knee Surg Sports Traumatol Arthrosc 2013;21:318-324.

9. Ward SR, Terk MR, Powers CM. Patella alta: association with patellofemoral alignment and changes in contact area during weight-bearing. J Bone Joint Surg Am 2007;89: 1749-1755.

10. Watson NA, Duchman KR, Grosland NM, Bollier MJ. Finite element analysis of patella alta: A patellofemoral instability model. Iowa Orthop J 2017;37:101-108.

11. Neyret P, Robinson AHN, Le Coultre B, Lapra C, Chambat P. Patellar tendon length-the factor in patellar instability? Knee 2002;9:3-6.

12. Greiwe RM, Saifi C, Ahmad CS, Gardner TR. Anatomy and biomechanics of patellar instability. Op Tech Sports Med 2010;18:62-67.

13. Lancourt JE, Cristini JA. Patella alta and patella infera. Their etiological role in patellar dislocation, chondromalacia, and apophysitis of the tibial tubercle. $J$ Bone Joint Surg Am 1975;57:1112-1115.

14. AL-Sayyad MJ, Cameron JC. Functional outcome after tibial tubercle transfer for the painful patella alta. Clin Orthop Relat Res 2002;(396):152-162.

15. Monk AP, Doll HA, Gibbons CLMH, et al. The pathoanatomy of patellofemoral subluxation. J Bone Joint Surg Br 2011;93:1341-1347.

16. Stefanik JJ, Zhu Y, Zumwalt AC, et al. Association between patella alta and the prevalence and worsening of structural features of patellofemoral joint osteoarthritis: the multicenter osteoarthritis study. Arthritis Care Res (Hoboken) 2010;62:1258-1265.

17. Blackburne J, Peel T. A new method of measuring patellar height. J Bone Joint Surg Br 1977;59:241-242.

18. Caton J, Deschamps G, Chambat P, Lerat JL, Dejour H. Patella infera. Apropos of 128 cases. Rev Chir Orthop Reparatrice Appar Mot 1982;68:317-325 [in French].

19. Insall J, Salvati E. Patella position in the normal knee joint. Radiology 1971;101:101-104.
20. Smith TO, Davies L, Toms AP, Hing CB, Donell ST. The reliability and validity of radiological assessment for patellar instability. A systematic review and meta-analysis. Skeletal Radiol 2011;40:399-414.

21. Yue R, Arendt E, Tompkins M. Patellar height measurements on radiograph and magnetic resonance imaging in patellar instability and control patients. J Knee Surg 2017;30:943-950.

22. Verhulst FV, van Sambeeck JDP, Olthuis GS, van der Ree J, Koëter S. Patellar height measurements: Insall-Salvati ratio is most reliable method. Knee Surg Sports Traumatol Arthrosc 2020;28:869-875.

23. Liu JN, Steinhaus ME, Kalbian IL, et al. Patellar instability management: A survey of the International Patellofemoral Study Group. Am J Sports Med 2018;46: 3299-3306.

24. Thévenin-Lemoine C, Ferrand M, Courvoisier A, Damsin J-P, Ducou le Pointe H, Vialle R. Is the CatonDeschamps Index a valuable ratio to investigate patellar height in children? J Bone Joint Surg Am 2011;93:e35.

25. Charles MD, Haloman S, Chen L, Ward SR, Fithian D, Afra R. Magnetic resonance imaging-based topographical differences between control and recurrent patellofemoral instability patients. Am J Sports Med 2013;41: 374-384.

26. Laugharne E, Bali N, Purushothamdas S, Almallah F, Kundra R. Variability of measurement of patellofemoral indices with knee flexion and quadriceps contraction: An MRI-based anatomical study. Knee Surg Relat Res 2016;28: 297-301.

27. Caton J. Method of measuring the height of the patella. Acta Orthop Belg 1989;55:385-386 [in French].

28. Askenberger M, Bengtsson Moström E, Ekström W, et al. Operative repair of medial patellofemoral ligament injury versus knee brace in children with an acute first-time traumatic patellar dislocation: A randomized controlled trial. Am J Sports Med 2018;46:2328-2340.

29. Barnett AJ, Prentice M, Mandalia V, Wakeley CJ, Eldridge JDJ. Patellar height measurement in trochlear dysplasia. Knee Surg Sports Traumatol Arthrosc 2009;17: 1412-1415.

30. Berg EE, Mason SL, Lucas MJ. Patellar height ratios: A comparison of four measurement methods. Am J Sports Med 1996;24:218-221.

31. van Duijvenbode D, Stavenuiter M, Burger B, van Dijke C, Spermon J, Hoozemans M. The reliability of four widely used patellar height ratios. Int Orthop 2016;40: 493-497.

32. Gracitelli GC, Pierami R, Tonelli TA, et al. Assessment of patellar height measurement methods from digital radiography. Rev Bras Ortop 2015:47:210-213.

33. Munch JL, Sullivan JP, Nguyen JT, et al. Patellar articular overlap on MRI Is a simple alternative to conventional measurements of patellar height. Orthop J Sports Med 2016;4:232596711665632.

34. Seil R, Müller B, Georg T, Kohn D, Rupp S. Reliability and interobserver variability in radiological patellar height ratios. Knee Surg Sports Traumatol Arthrosc 2000;8:231-236. 
35. Smith TO, Cogan A, Patel S, Shakokani M, Toms AP, Donell ST. The intra- and inter-rater reliability of X-ray radiological measurements for patellar instability. Knee 2013;20:133-138.

36. Grelsamer RP, Meadows S. The modified Insall-Salvati ratio for assessment of patellar height. Clin Orthop Relat Res 1992;282:170-176.

37. Scuderi GR, Windsor RE, Insall JN. Observations on patellar height after proximal tibial osteotomy. J Bone Joint Surg Am 1989;71:245-248.

38. Biedert RM, Albrecht S. The patellotrochlear index: a new index for assessing patellar height. Knee Surg Sports Traumatol Arthrosc 2006;14:707-712.
39. Dejour D, Ferrua P, Ntagiopoulos PG, et al. The introduction of a new MRI index to evaluate sagittal patellofemoral engagement. Orthop Traumatol Surg Res 2013;99:S391-398 (8 suppl).

40. Shabshin N, Schweitzer M, Morrison W, Parker L. MRI criteria for patella alta and baja. Skeletal Radiol 2004;33.

41. Lee PP, Chalian M, Carrino JA, Eng J, Chhabra A. Multimodality correlations of patellar height measurement on X-ray, CT, and MRI. Skeletal Radiol 2012;41: 1309-1314.

42. Miller TT, Staron RB, Feldman F. Patellar height on sagittal MR imaging of the knee. AJR Am J Roentgenol 1996;167:339-341. 